\title{
Design and Analysis of Computer Experiments - An Application to a Complex Industrial Component
}

\author{
PIETRO TARANTINO ${ }^{1}$, ARMANDO STAGLIANO $^{1}$, MAGNUS ARNÉR $^{2}$ \\ ${ }^{1}$ Tetra Pak Packaging Solutions, via Delfini 1, 41100, Modena, Italy \\ ${ }^{2}$ Tetra Pak Packaging Solutions, Ruben Rausings gata, 22186 Lund, Sweden
}

\begin{abstract}
Since the formal introduction of computer experiments back in 1989, substantial work has been done to make these experiments as efficient and effective as possible. As a consequence, more and more industrial studies are performed. In this direction, sequential strategies have been introduced with the aim of reducing the experimental effort while keeping the required accuracy of the meta-model. The strategies consist in building a fairly accurate meta-model based on a low number of experimental points, and then adding new points in an iterative way according to some strategy like improving the accuracy of meta-model itself or finding the optimal design point in the design space. In this work, a hybrid of these two strategies is used, with the aim to achieve both meta-model accuracy and optimum design solution while keeping low the experimental effort. The proposed methodology is applied to an industrial case study. The pragmatism of such hybrid strategy, together with simplicity of implementation promotes the generalization of this approach to other industrial experiments.
\end{abstract}

Key Words: Computer Experiments, Space-Filling Designs, Kriging, Sequential Strategy, Design Optimization.

\section{Introduction}

Experimentation is an integral part of any development process (Wu and Hamada, 2000). Traditionally, this experimentation has been limited to physical tests. The nature of these experiments, e.g. costly, time-consuming and difficult to set, usually forces experimenter to test a small number of factors at few levels. Typically, issues such as replication, randomization and blocking have to be addressed (Vicario, 2006, Arnér, 2014). In computer experiments, neither randomization nor replication is needed (and mostly not even blocking), and a large number of factor levels is fairly easy to handle. In general, the time and the cost of the study can be reduced (Santner et al. 2003).

Since the formal introduction of computer experiments by Sacks et al. (1989), substantial work has been done to make these experiments as efficient and effective as possible and, as a consequence, more and more industrial studies are performed by replacing physical experimentation with a "virtual" one, in which a computer runs a program that simulates the behaviour of the system of reference (Fang et al. 2006).

There is not a unique approach to design and analysis of computer experiments (Chen et al. 2003). Traditionally, space filling or optimal designs have been used for exploring the design region while polynomial regressions and kriging models have been extensively used to build the meta-model or emulator.

Since the seminal article by Sacks et al (1989), sequential strategies have been introduced with the aim of reducing the experimental effort while keeping the required accuracy from the experiment (e.g. Ascione et al., 
2012). They consist of building fairly accurate meta-models based on a small number of experimental points, and then adding new points in an iterative way by updating the meta-model each time a new data point is added. The new data point is selected according to a strategy, e.g. aiming at improving the accuracy of meta-model itself or finding the optimal design point in the design space.

In this work, a hybrid approach to the sequential approach based on the work of Bonte et al. (2010) is applied. It is built with the targetto achieve both meta-model accuracy and optimum design solution while keeping the experimental effort low. The proposed methodology is applied to an industrial case study. The pragmatism of such hybrid strategy, together with simplicity of implementation promotes the generalization of this approach to other industrial experiments.

In section 2, we will give a short introduction to computer experiments in general and sequential strategies in particular. We will then present the proposed hybrid approach in a schematic way in section 3 and its application to a sealing component optimization in section 4. As a part of the conclusions, we reflect on the current and future role of computer experiments in industrial applications and we propose hints for future works.

\section{Sequential Strategies for Computer Experiments}

Running a simulation model on a computer is often time consuming. One single run may take hours or even days. If some changes of parameters are required (for example material parameters or boundary conditions), the new run has a tendency to take an equally long time. This makes it time consuming to optimize some property of the design or,for example, to explore the robustness of the design to variation. As a way to partly overcome this problem, the concept of meta-models has emerged.

Meta-model means "model of a model" (Mohanty, 2015). Starting from the definition of the design space, i.e. the domain within which the designer has the freedom to select the design factor values, the response is calculated by computer simulations (based on a model of reality) at different locations of the design space. With these points, statistical methods are used to build a simplified mathematical model, a so-called meta-model, to approximate the response over the design space. This model is then used as the "true" model. A meta-model can be used for a wide variety of applications, such as exploring the robustness, optimizing some property, or even to address questions not yet asked at the time of building the meta-model. Since the calculation time for a meta-model usually is small, the gain in simulation time may be substantial. In this paper, we will use the meta-model for optimization purposes.

In a typical approach to design optimization by Computer Experiments (see Figure 1) the meta-model is used to find the optimal design point, and finally this optimal point is verified by a new computer simulation using the original model. Thus, the original model of reality is only used in the first stage, to generate data and build the meta-model, and in the stage of verification.

In order to build a good meta-model, computer simulations are performed according to some experimental design, typically a space-filling design (Bursztyn and Steinberg, 2006). In a space-filling experimental design, points are spread out as much as possible in the design space in order to achieve information about the entire region (Johnson et al., 2010).

A Latin Hypercube Design (LHD) is a popular type of space-filling design for computer experiments. There is not unique LHD in a design space using the same amount of points. A criterion of selection is typically used to choose the LHD which best suits the designer needs (Silvestrini et al., 2013). In this work the "maximin" 


\section{Complex Industrial Component}

LHD is used. A space filling design is optimal with respect to the maximin criterion if the minimal distance between any pair of design points is maximized. Thus, the points are spread out as much as possible in the design space.

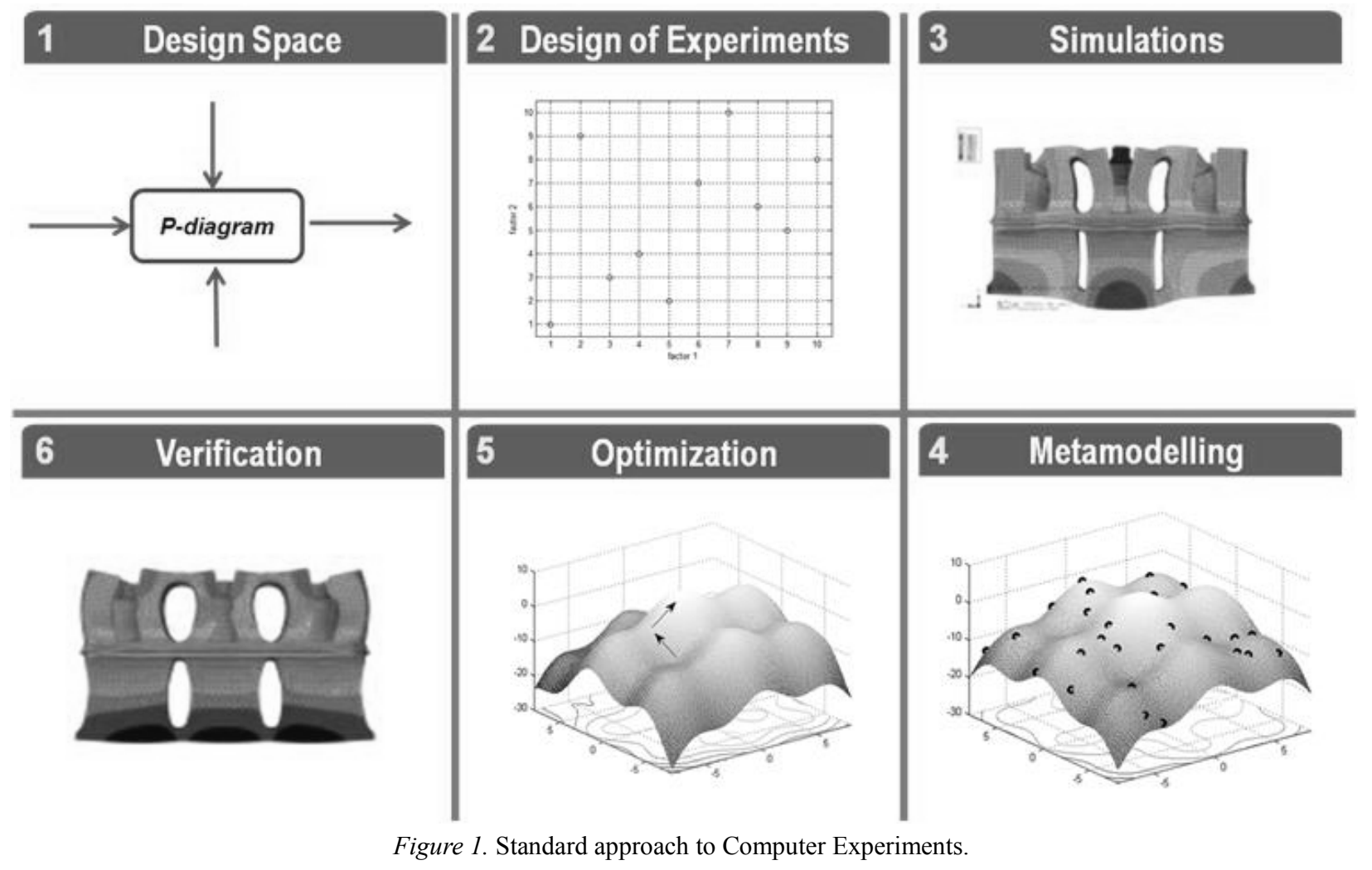

There are many different statistical techniques that can be used to build a meta-model, such as various kinds of regression analysis (Jin et al. 2001). Another popular technique is kriging (Cressie, 1993), and it is the approach that is used in this paper. This technique provides two important outputs: the kriging mean, which interpolates the data and it is typically used for prediction, and the kriging variance (or standard deviation), which is zero at the data points and increases with the distance from them, so giving indication of prediction uncertainty.

The meta-model is an approximation of the original computer model, and its accuracy is crucial for a good design optimization. The quality of the meta-model is proportional to the number of data points used to build it: the more the points, the more accurate the meta-model. On the other hand, it is clear that with an increased number of points, the required time to perform all the computer simulations and build the meta-model is also increased. A good compromise between meta-model quality and computational time can be a sequential strategy where an initial design for computer experiments is used to build an initial meta-model, and then new design points are added, one after each other, until the meta-model has reached the expected accuracy and/or the design optimum is found (Gramacy and Lee, 2009). Once a new point is selected and simulated, it is added to the existing data set for a new meta-model construction. At every new iteration, the meta-model is based on one more data point and so it is more accurate, in particular in the region close to the new data point. The iterations finally stop according to some criterion set by the user. The approach used to iteratively select new 


\section{Complex Industrial Component}

data points is fundamental for the efficiency of the sequential strategy, and it depends mainly on the purpose of the user.

For the rest of the paper, the following symbols are used:

$$
\begin{gathered}
\boldsymbol{x} \leftarrow \text { design point; } \\
y(\boldsymbol{x}) \leftarrow \text { response calculated by simulation; } \\
\hat{y}_{N}(\boldsymbol{x}) \leftarrow \text { metamodel prediction (kriging mean) at the Nth iteration; } \\
\hat{s}_{N}(\boldsymbol{x}) \leftarrow \text { prediction uncertainty (kriging standard dev. ) at the Nth iteration; } \\
\boldsymbol{x}_{\text {opt }, N} \leftarrow \text { optimal point at the Nth iteration. } \\
\boldsymbol{x}_{N} \leftarrow \text { point added at the Nth iteration. }
\end{gathered}
$$

There are three main sequential strategies which can be implemented according to Bonte et al. (2010):

Informative Criterion (IC) - New data points are added where there is the highest lack of information in the design space. In case of kriging, the new point can be selected where the kriging standard deviation takes its maximum

$$
\boldsymbol{x}_{N} \text { is selected so that } \hat{s}_{N-1}\left(\boldsymbol{x}_{\boldsymbol{N}}\right)=\max \left\{\hat{s}_{N-1}(\boldsymbol{x})\right\}
$$

This procedure is typically repeated until the meta-model accuracy is good enough with respect to some convergence criterion.

Optimization Criterion (OC) - The new data point is selected where the meta-model predicts the optimum. For example, if the goal is to maximize the response, the new data point is selected where meta-model predicts the maximum response. In case of kriging, the new point is selected where kriging mean takes the following:

$\boldsymbol{x}_{\text {opt }, N}$ is the point such that $\hat{y}_{N-1}\left(\boldsymbol{x}_{\text {opt }, N}\right)=\max \left\{\hat{y}_{N-1}(\boldsymbol{x})\right\}$ in a maximization problem,

$\boldsymbol{x}_{\text {opt }, N}$ is the point such that $\hat{y}_{N-1}\left(\boldsymbol{x}_{\text {opt }, N}\right)=\min \left\{\hat{y}_{N-1}(\boldsymbol{x})\right\}$ in a minimization problem.

This procedure is typically repeated until the new optimum is already in the data set or close to one in the data set.

Before describing the third criterion, it is useful to highlight some benefits and limitations of IC and OC. The Informative Criterion is good to improve meta-model accuracy over the design space, but can be inefficient if the final purpose is design optimization. There can be regions in the design space where the meta-model accuracy is bad, but the response is far from the optimum and the user is not interested to explore it more in depth. Applying the IC strategy, new data points may be added in this bad design region, causing useless computational effort. The Optimizing Criterion typically converges very fast to the design optimum, but it does not take the meta-model accuracy into consideration. According to the OC, the new design point is selected where the optimum is predicted, but if the meta-model accuracy is bad, the risk to fall in a local or false optimum is high. In order to overcome these limitations, a third strategy can be considered.

Mixed Criterion (MC) - The new data point is selected by considering a weighted sum of meta-model prediction and prediction uncertainty.

$$
\begin{gathered}
\qquad \leftarrow \text { weight factor } \\
f_{\text {merit }, N}(\boldsymbol{x})=\hat{y}_{N}(\boldsymbol{x}) \pm k \cdot \hat{s}_{N}(\boldsymbol{x}) \leftarrow \text { merit function } \\
\boldsymbol{x}_{\text {opt }, N} \text { is the point that maximizes } \hat{y}_{N-1}(\boldsymbol{x})+k \cdot \hat{s}_{N-1}(\boldsymbol{x}) \\
\boldsymbol{x}_{\text {opt }, N} \text { is the point that minimizes } \hat{y}_{N-1}(\boldsymbol{x})-k \cdot \hat{s}_{N-1}(\boldsymbol{x})
\end{gathered}
$$

According to this approach, a new data point is selected where there is a good predicted response, but not necessarily the predicted optimum, as well as a large uncertainty that needs to be reduced. Of course, if the 


\section{Complex Industrial Component}

weight factor $k$ is large, more importance is given to the uncertainty with respect to the prediction, and vice versa when $k$ is smaller:

$$
\begin{aligned}
& k \rightarrow 0, M C \rightarrow O C \\
& k \rightarrow \infty, M C \rightarrow I C
\end{aligned}
$$

Moreover, larger $k$ implies more iteration to converge to the final meta-model. $k=1$ is often a selected compromise, also chosen in this work. The typical behaviour of the Mixed Criterion is the following: at the beginning, meta-model accuracy is low so $\hat{s}_{N}(\boldsymbol{x})$ prevails on $\hat{y}_{N}(\boldsymbol{x})$ in the merit function and $M C \rightarrow I C$, during the last iterations accuracy is good, $\hat{y}_{N}(\boldsymbol{x})$ prevails on $\hat{s}_{N}(\boldsymbol{x})$ so $M C \rightarrow O C$. Iterations can be stopped when the new selected point is already in the data set. Note that if kriging technique is used, the merit function lies on the boundary of a Gaussian process confidence interval, whose width depends on $k$.

An illustrative example of these three sequential strategies is shown in Figure 2. Table 1, instead, summarizes benefits and limitations of each sequential strategy.

In the rest of the paper, the optimum predicted by the meta-model will be called "predicted optimum", and the optimum of the merit function (according to the mixed criterion) will be called the "potential optimum". "Potential optimum" means that, even if it is not the optimum predicted by the meta-model, due to the higher prediction uncertainty the response at this design point can be even potentially better.

Table 1

Sequential strategies: benefits and limitations

\begin{tabular}{|l|l|l|}
\hline Sequential Strategies & Benefits & Limitations \\
\hline Informative & The global accuracy of the meta-model is improved & $\begin{array}{l}\text { New data points could be selected also in } \\
\text { bad design regions that are of low interest } \\
\text { for deeper exploration }\end{array}$ \\
\hline Optimization & $\begin{array}{l}\text { It converges very quickly, and new data points are selected } \\
\text { only at locations of interest for deeper exploration }\end{array}$ & $\begin{array}{l}\text { High risk to fall in a local optimum if the } \\
\text { meta-model is not enough accurate. }\end{array}$ \\
\hline $\begin{array}{l}\text { New data points are added only at locations we are } \\
\text { interested to explore more deeply, but with a smaller risk } \\
\text { than OC to fall in a local optimum since the prediction } \\
\text { uncertainty is also taken into account. }\end{array}$ & $\begin{array}{l}\text { It can take time to converge if the } \\
\text { uncertainty and/or the weight factor } k \text { are } \\
\text { high (too many potential optima are found). }\end{array}$ \\
\hline
\end{tabular}

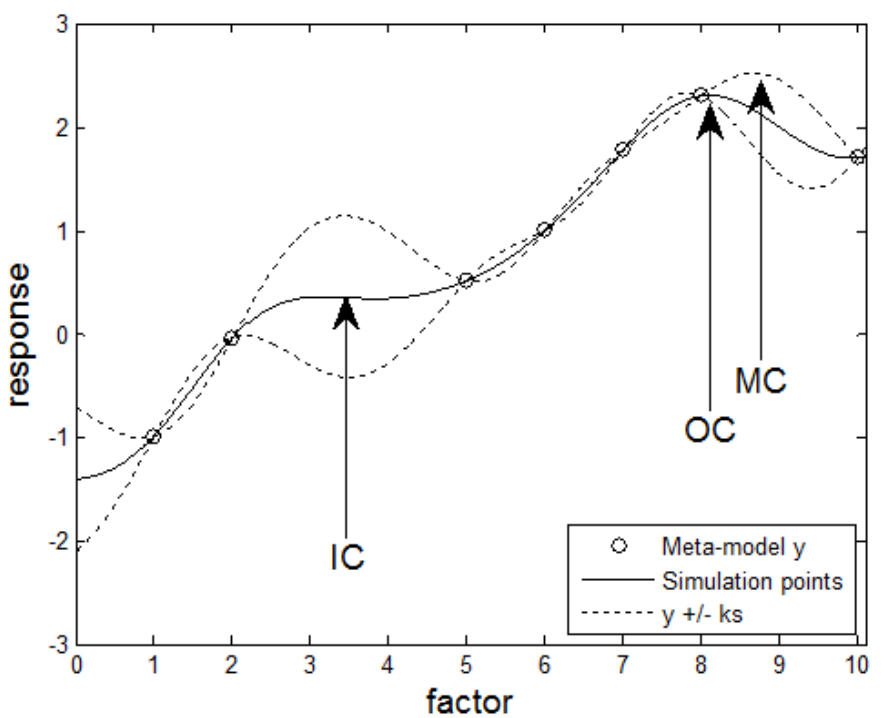

Figure 2. Sequential strategies: in this example, the new point is selected where the merit function is maximum. Based on a figure in Bonte et al. (2010). 
Complex Industrial Component

\section{Proposed sequential and pragmatic approach}

The proposed sequential approach is illustrated in Figure 3 and described below. It can be divided into two main steps: initialization and sequential strategy.

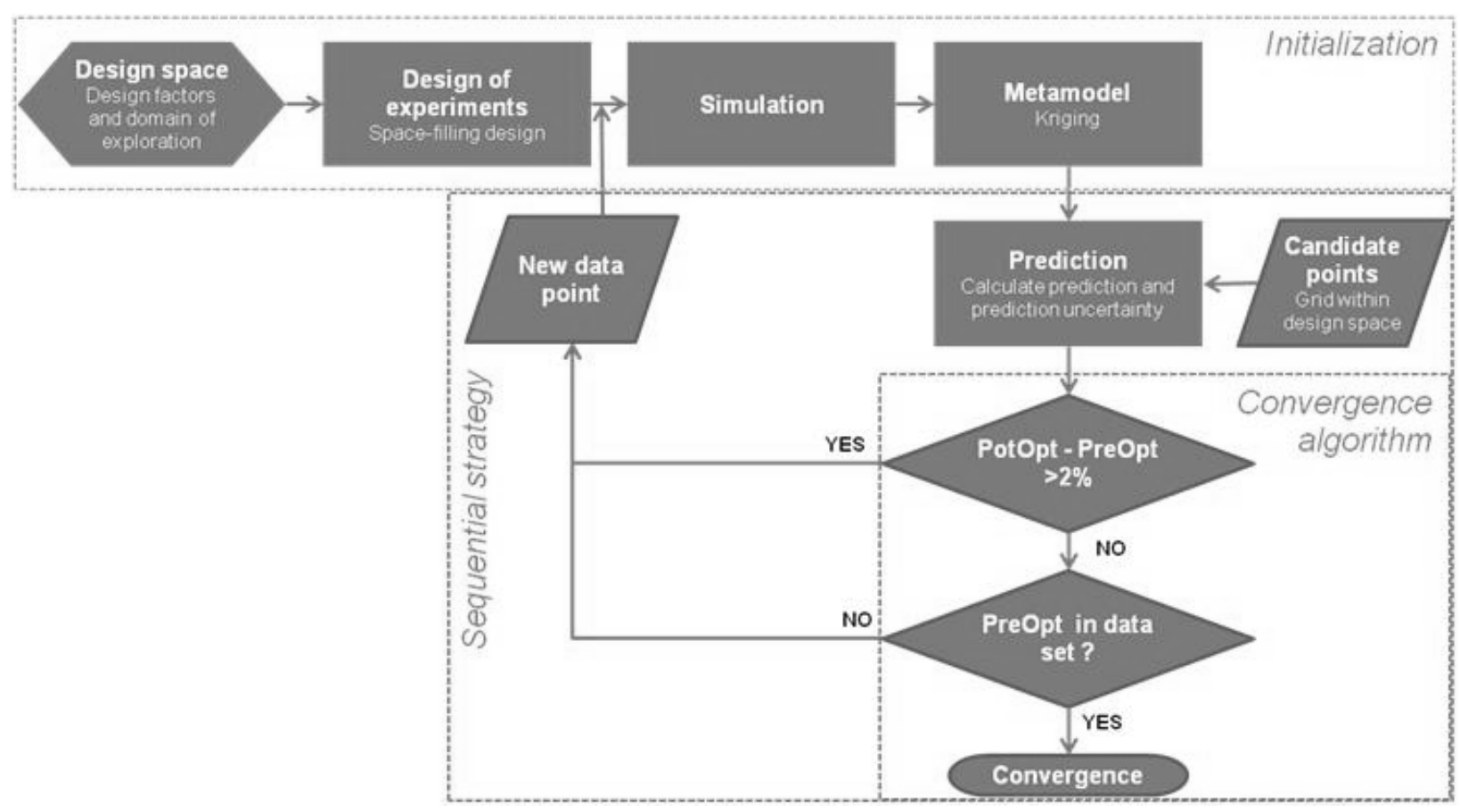

Figure 3. Proposed sequential approach.

\section{Initialization}

Design space: the design factors and their domain of variation are defined;

Design of Experiment: a maximin LHD is defined. In this stage of initialization is not convenient to define too many experimental points. On the other hand, with a too small number of initial points, some peaks and valleys may be missed and the risk to get stuck in local optimum is higher. Our recommendation is to follow the well-know rule of thumb of $10 \times m$ points, where $m$ is the number of design factors (Loeppky et al. 2009).

Simulation: the response is calculated at each design point by computer simulations.

Meta-model: a kriging meta-model is built using the design points and corresponding responses.

\section{Sequential strategy}

Candidate points: a grid of points, as dense as possible, is defined in the design space. In this work, a grid of $10^{m}$ candidate points for the $m$-dimensional design space was defined.

Prediction: The kriging mean and standard deviation are calculated at each point of the grid.

Predicted optimum vs. potential optimum: two optimal points are found among the grid points, the predicted optimum and the potential optimum. As previously described, "potential optimum" means that the actual response at this location could be potentially even better when considering a large prediction uncertainty. If the potential optimum value is close to the predicted optimum value, it is likely that the predicted optimum is close the true rather than a local optimum, so MC is switched to OC to speed up the convergence. Therefore, 


\section{Complex Industrial Component}

only the predicted optimum is taken into account. Here it is suggested to switch from MC to OC when the difference between predicted and potential optimum is less than $2 \%$. To summarize, if the difference is bigger than $2 \%$, then it is taken the potential optimum, otherwise the predicted optimum.

If MC has switched to OC, the fulfilment of the convergence criterion is verified. The criterion used in this paper is thatthe sequential addition of points stops if the new optimal point is already in the data set.

If the answer is $\mathrm{NO}$, the optimum is added to the data set and steps from 3 to 7 are repeated again.

If the answer is YES, the strategy has finally converged to the design optimum.

\section{Case study: sonotrode optimization}

\section{Problem description}

Tetra Pak is a multinational company that supply complete systems for processing, packaging and distributions of liquid food products. Tetra Pak portfolio includes many different packages such as theaseptic packages, that allow liquid food quality to be retained for a long time without the need for preservatives or refrigeration. The principle of aseptic packaging is sketched in Figure 4. Flat and unformed packaging material passes through a heated hydrogen peroxide bath that heats the material during a given period of time. Hydrogen peroxide is then eliminated from the packaging material using pressure rollers or hot air. The sterilized packaging material is then maintained in a closed sterile environment where it is filled with UHT (Ultra High Temperature) treated food, sealed longitudinally and transversally and finally folded into the final shape.

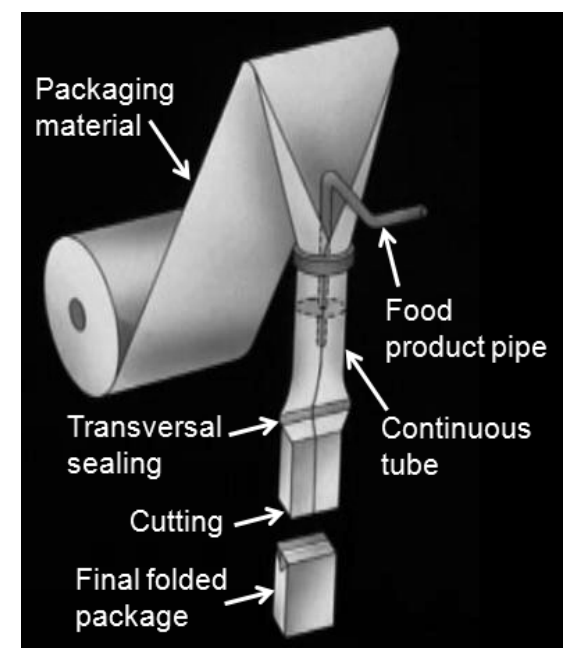

Figure 4. Principle of aseptic packaging.

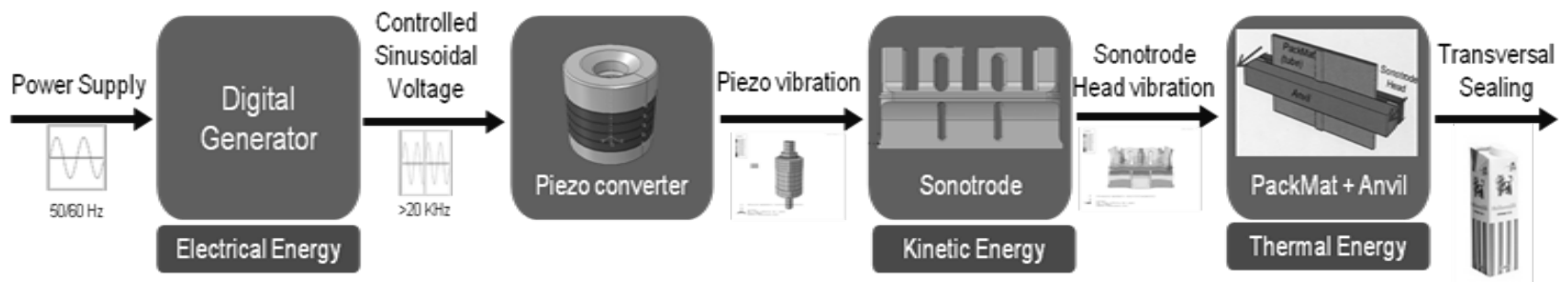

Figure 5. Ultrasonic sealing device.

The transversal sealing of packaging material can be obtained in different ways, depending on different factors such as the specific product to be filled and the packaging material to be used. In some cases, 


\section{Complex Industrial Component}

transversal sealing is performed by using an ultrasonic device which is functionally described into figure 5. A standard electrical supply is transformed into an ultrasonic frequency range $(20 \mathrm{kHz}-1 \mathrm{GHz})$ by means of a generator. This energy is transferred to piezo-electric elements that start to vibrate causing the oscillation of the head of a mechanical component where they are mounted, called sonotrode. Finally, the surface of the head presses the package material against an anvil, causing heat generation and, as a consequence, the sealing of the material.For a good package sealing, the sealing surface of the sonotrode must remain as flat as possible during the oscillation.

In Figure 6 the deformation of a reference sonotrode during ultrasonic frequency oscillation, calculated by FEM modal analysis, is shown. The objective of this work is to find, by computer simulations and metamodeling technique, the optimal design of the sonotrode which ensures the highest possible flatness of the sealing area during ultrasonic oscillation. In particular, the objective is to minimize the standard deviation of the sealing area nodal displacements.

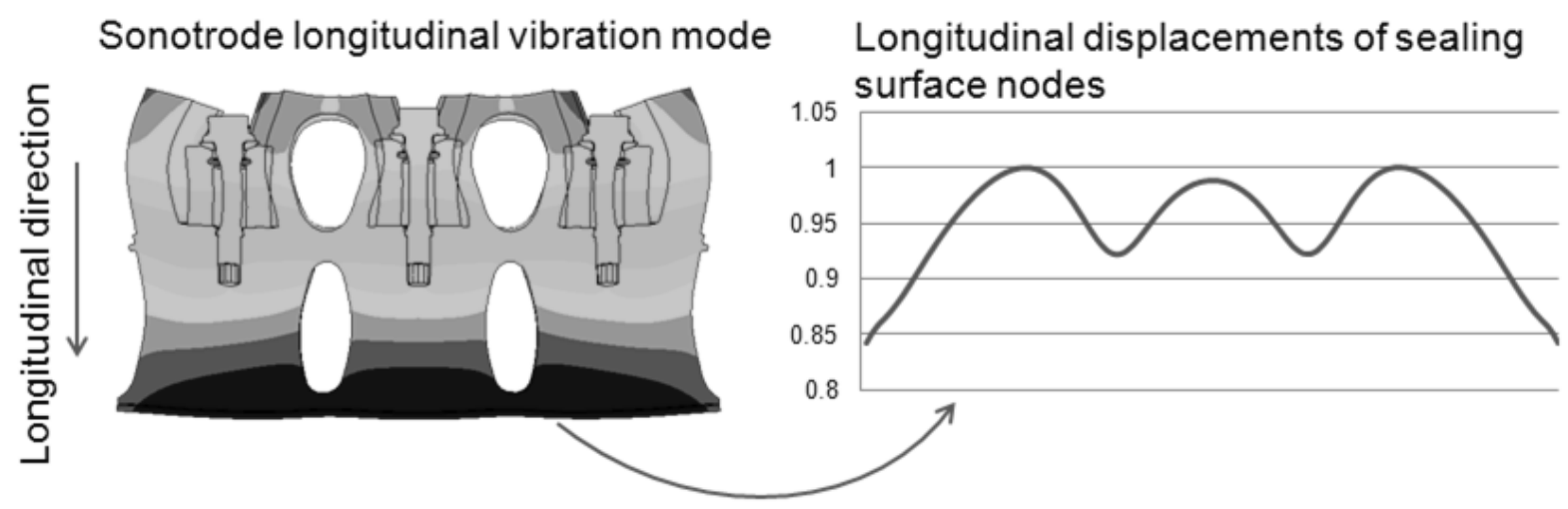

Figure 6. Sonotrode sealing area oscillation by FEM analysis.

Sealing area flatness depends on the geometry of the sonotrode. Six design factors were selected after a brainstorming session with subject-matter experts (see Figure 7 and Table 2). The domain of exploration of the design factors was defined according to design feasibility. However, for confidentiality purpose the range of values is not here presented.

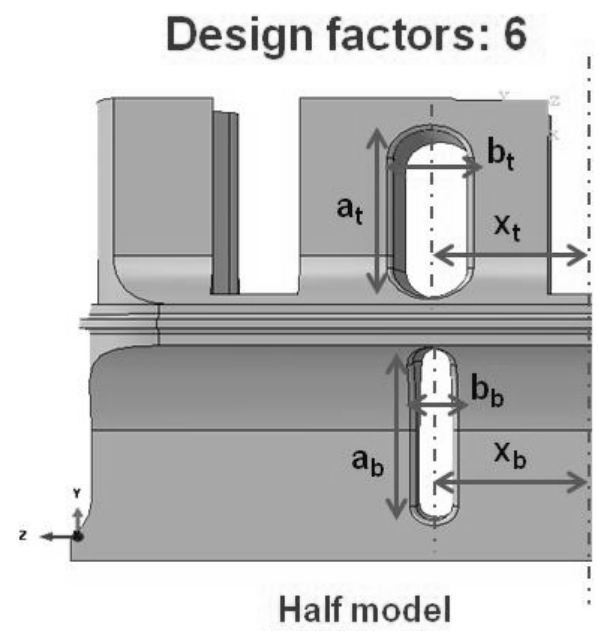

Figure 7. Design factors. 
Table 2

Design factors definition

\begin{tabular}{ll}
\hline Symbol & Definition \\
\hline $\mathrm{x}_{\mathrm{t}}$ & Top hole centre horizontal position \\
$\mathrm{b}_{\mathrm{t}}$ & Top hole minor dimension \\
$\mathrm{a}_{\mathrm{t}}$ & Top hole major dimension \\
$\mathrm{x}_{\mathrm{b}}$ & Bottom hole centre horizontal position \\
$\mathrm{b}_{\mathrm{b}}$ & Bottom hole minor dimension \\
$\mathrm{a}_{\mathrm{b}}$ & Bottom hole major dimension \\
\hline
\end{tabular}

\section{Design optimization}

The design optimization was carried out using three different software tools:

- Dassault Systems Abaqus, for FEM analysis;

- Dassault Systems Isight, for the optimization workflow;

- $\mathrm{R}$, for statistical computing.

The optimization workflow in Isight environment is shown in Figure 8; it follows the procedure illustrated in Section 3 and it is divided in two main steps: initialization and sequential strategy.

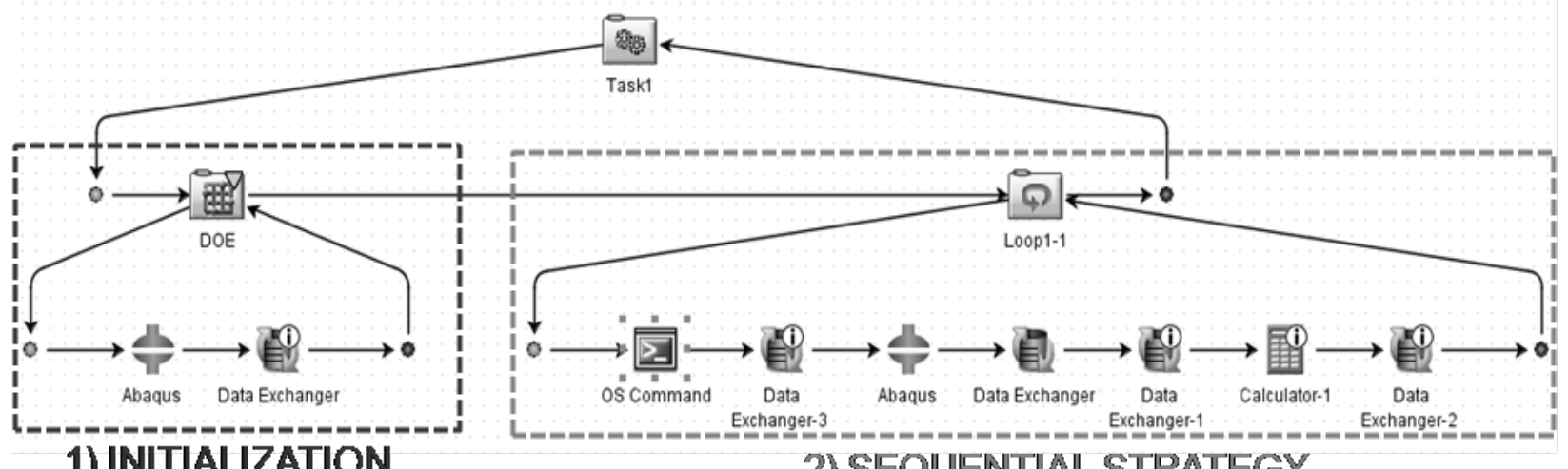

1) INITIALIZATION

2) SEQUENTTIAL STRRATEGY

Figure 8. Optimization workflow.

During initialization, data points for the initial meta-model are calculated. A 60-points maximin LHD, and the response corresponding to each design point calculated by computer simulation. Finally, the list of data points (design points and corresponding responses) is recorded in a text file (Data Exchanger module).

The sequential strategy consists of an iterative loop. Through the Operative System (OS) Command, it is possible to send the list of data points to $\mathrm{R}$ for the meta-model estimation. Always in $\mathrm{R}$, the optimum according to the proposed sequential approach is found, and its coordinates in the design space are saved in another text file. This text file is then opened by Isight, and it is checked whether the design point is already in the data set; if not, the design coordinates are sent to the computer simulator (Abaqus) to calculate the response and then added to the data set for a new iteration. Otherwise, if the optimum is already in the data set, the loop is stopped.

In this case study, the sonotrode design optimization was carried out by 176 runs (Table 3): 60 runs for the initialization and 116 runs for the sequential strategy iterations. 
Table 3

Sonotrode design optimization: number of runs and time

\begin{tabular}{lll}
\hline & Number of runs & Computational time \\
\hline Initialization & 60 & $11 \mathrm{~m}$ \\
Sequential strategy & 116 & $21 \mathrm{~h} 16 \mathrm{~m}$ \\
Total & 176 & $32 \mathrm{~h} 16 \mathrm{~m}$ \\
\hline
\end{tabular}

The evolution of the sequential optimization is shown in Figure 11. For simplicity, some symbols introduced in Section 2 are resumed here.

$\hat{y}_{N}\left(\boldsymbol{x}_{N}\right)$ : Kriging mean (i.e., prediction) at the optimal point at the $N$ th iteration.

$\hat{s}_{N}\left(\boldsymbol{x}_{N}\right)$ : Kriging standard deviation (i.e., prediction uncertainty) at the optimal point at the $N$ th iteration.

$\hat{y}_{N}\left(\boldsymbol{x}_{N}\right)+\hat{s}_{N}\left(\boldsymbol{x}_{N}\right)$ : Merit function (with $k=1$ ) at the optimal point at the $N$ th iteration.

$y\left(\boldsymbol{x}_{N}\right)$ : Response calculated by simulation at the optimal point at the $N$ th iteration.

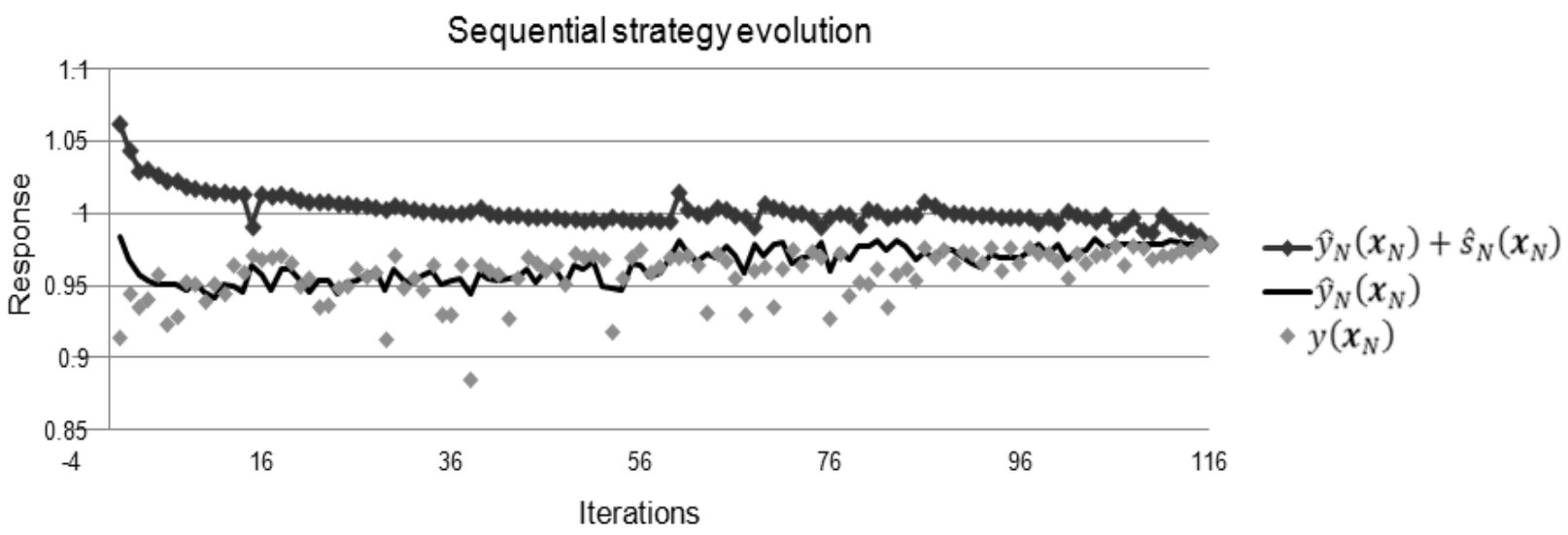

Figure 9. Sequential optimization evolution.

The response is, as previously described, the standard deviation of the sonotrode sealing area nodal displacements. It can be observed, from Figure 9, that the merit function, prediction and simulation are very different during the first iterations. The meta-model accuracy is not good, $\hat{s}_{N}\left(\boldsymbol{x}_{N}\right)$ is high as well as the difference between the merit function and the prediction. Also the difference between the prediction and simulation result at the optimal point is high, confirming that meta-model accuracy is low. When $\hat{s}_{N}(\boldsymbol{x})$ is high, the Mixed Criterion works in a similar way to the Informative Criterion. Going on with the iterations, $\hat{s}_{N}\left(\boldsymbol{x}_{N}\right)$ becomes smaller as well as the difference among merit function, prediction, and simulation. When $\hat{s}_{N}(\boldsymbol{x})$ is small, the Mixed Criterion works in a similar way to the Optimizing Criterion. Only at the last iteration merit function, prediction, and simulation are equal: the optimum is already in the data set, so $\hat{s}_{N}\left(\boldsymbol{x}_{N}\right)=0$ and the meta-model interpolates the response calculated by the simulation in that point.

Sealing area displacements of the optimal and actual reference sonotrode are compared in Figure 10 and Table 4. It is quite clear that the optimal solution shows larger and flatter sealing area displacements with respect to reference solution, in particular the standard deviation is about $1 / 3$ smaller. 


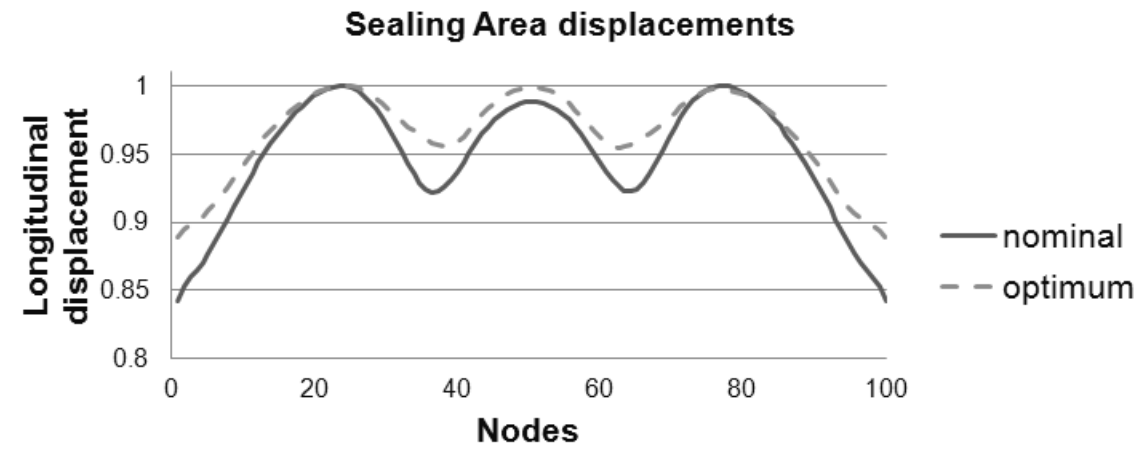

Figure 10. Sealing area displacement: actual vs. optimum.

Table 4

Reference vs. Optimal design

\begin{tabular}{lll}
\hline Sealing area displacement & Reference design & Optimal design \\
\hline Standard deviation & $100 \%$ & $64.1 \%$ \\
\hline
\end{tabular}

\section{Conclusions \& Discussion}

The role of computer experiments in industrial applications is becoming as important as the one of physical experiments. There are several reasons that might explain this trend, for instance an increased awareness on statistics in general and computer experiments in particular among the development engineers community, statistical methods embedded into simulation software and some clear advantage on project time and cost. A hybrid sequential approach to optimization via computer experiment has been described showing in concrete - how it is possible to achieve both meta-model accuracy and optimum design solution while keeping the experimental effort as low as possible. The approach has been implemented into a unique work environment, making the entire sequential procedure fully automatic and showing very interesting linking between a simulation environment like Dassault Systems Isight and a statistical software like R. The pragmatism of such a strategy, together with simplicity of implementation promotes the generalization of this approach to other industrial experiments.

Three research streamsto be further developed, could speed up even more the application of computer experiments in the industrial arena, namely:

- Methodologies for screening phase in case of high number of design factors and complex relationship between factors and response (see, for example, Linkletter et al. 2006)

- Multi-objective or multi-constraint optimization in case of highly complex engineering problems (see, for example, Steenackers et al. 2008)

- Robust design via Computer Experiments in case the variability of the usage environment is included into the design phase (see, for example, Giovagnoli and Romano, 2008)

Two additional aspects of this work may be worth mentioning since it may affect future work of similar type. One concerns the fact that we pre-defined a set of candidate points (see section 5, point 5). This choice had, e.g., an effect on our convergence criteria and is closely linked to it in the sense that the closer the grid of candidate points, the more demanding is the convergence criteria. An alternative approach would have been to allow any point within the design region and have a convergence criterion that the new point is on a distance 


\section{Complex Industrial Component}

$<\varepsilon$ from an existing point; the density of the grid and the value of $\varepsilon$ play the same role.

Another aspect concerns the intended usage of the meta-model. In our case it was optimization. However, there are several other possible purposes. For some of them, knowledge of the system behaviour in 'bad' points may be of larger interest than in optimization. If the same type of algorithm as here, the Mixed Criterion, is used, is may require a large value of $k$ in the merit function.

\section{Acknowledgment}

The authors gratefully acknowledge the support of Tetra Pak Systems Engineering Department. Specifically, thanks to Alberto Mameli for his interest in statistical methods and support in the simulation model. The authors would like to thank also Luca Gentili from Sealing Department for the support in the ultrasonic sealing process understanding and optimization results' analysis.

\section{References}

Arnér M. Statistical Robust Design. An Industrial Perspective. Wiley, Chichester, 2014.

Ascione R, Moroni G, Petro' S, Romano D. Adaptive Inspection in coordinate metrology based on Kriging models. Precision Engineers 2012; 37(1): 44-60.

Bonte MHA, Fourment L, Do T, Van den Boogaard AH, Huetink J. Optimization of forging processes using Finite Element simulations. Structural Multidisciplinary Optimization 2010; 42: 797-810.

Bursztyn D, Steinberg DM. Comparison of designs for computer experiments. Journal of Statistical Planning and Inference 2006; 136: 1103-1119.

Chen VCP, Tsui KL, Barton RR, Allen JK. A review of design and modeling in computer experiments. In: Handbook of Statistics, vol. 22. Elsevier: Amsterdam, 2003; 231-261.

Cressie NAC. Statistics for Spatial data. Wiley, Chichester, 1993.

Fang KT, Li R, Sudjanto A. Design and Modelling for Computer Experiments. Chapman Hall/CRC:London, 2006.

Giovagnoli A, Romano D. Robust Design via Simulation Experiments: A Modified Dual Response Surface Approach. Quality \& Reliability Engineering International 2008; 24: 401-416.

Gramacy RB, Lee HKH. Adaptive Design and Analysis of Supercomputer Experiments. Technometrics 2009; 51(2): $130-145$.

Jin R, Chen W, Simpson TW. Comparative studies of metamodelling techniques under multiple modelling criteria. Structural and Multidisciplinary Optimization 2001; 23: 1-13.

Johnson RC, Montgomery DC, Jones B, Parker PA. Comparing Computer Experiments for Fitting High-Order Polynomial Metamodels. Journal of Quality Technology 2010; 42(1): 86-102.

Linkletter C, Bingham D, Hengartner N, Higdon D. Variable Selection for Gaussian Process Models in Computer Experiments. Technometrics 2006; 48(4): 478-490.

Loeppky JL, Sacks J, Welch W. Choosing the sample size of a computer experiment: a practical guideline. Technometrics 2009; 48: 478-490.

Mohanty SP. Nanoelectronic. Mixed-Signal System Design. McGraw-Hill, 2015.

Sacks J, Welch WJ, Mitchell TJ, Wynn HP. Design and Analysis of Computer Experiments. Statistical Science 1989; 4(4): 409-435.

Santner TJ, Brian JW, Notz IW. The Design and Analysis of Computer Experiments. Springer: New York, 2003.

Silvestrini RC, Montgomery DC, Jones B. Comparing Computer Experiments for the Gaussian Process Model Using Integrated Prediction Variance. Quality Engineering 2013; 25(2): 164-174.

Steenackers G, Guillaume P, Vanlanduit S. Robust Optimization of an Airplane Component Taking into Account the Uncertainty of the Design Parameters. Quality \& Reliability Engineering International 2008; 25 (3): 255-282.

Vicario G. Modelling and Analysis of Physical and Computer Experiments. Quality and Reliability Engineering International 20065; 22(5): 503-505.

Wu CFJ, Hamada M. Experiments: Planning, Analysis, and Parameter Design Optimization. Wiley: New York, 2009. 
About the authors

Pietro Tarantino received a Ph.D. in Total Quality Management from the University of Naples "Federico II" (Italy). He is author of several international publications in the field of statistical methodologies for product development. Currently, he is a Manager in the area of Quality at Tetra Pak Packaging Solutions, Italy. His e-mail address is: pietro.tarantino@tetrapak.com

Armando Stagliano is an aerospace engineer, graduated at the University of Naples "Federico II" (Italy) with a master thesis on Computer Experiments. Currently, he works in the area of Design and Development at Tetra Pak Packaging Solutions, Italy. His e-mail address is: armando.stagliano@tetrapak.com

Magnus Arnér received a M Sc in Industrial Engineering in 1988 and a Lic Eng in mathematical Statistics in 1992, both at Linköping Institute of Technology (Sweden). He has worked with application of engineering statistics in the industry since the 1990'ies and is the author of the book "Statistical Robust Design, An Industrial Perspective". He is currently working with development at Tetra Pak Packaging Solutions in Sweden. magnus.arner@tetrapak.com 